\title{
Planar-fingerprint transition in a thermoreversible liquid crystalline gel
}

\author{
Alberto de Lózar, Wolfgang Schöpf, ${ }^{*}$ and Ingo Rehberg ${ }^{\dagger}$ \\ Experimentalphysik V, Universität Bayreuth, D-95440 Bayreuth, Germany \\ Oscar Lafuente and Günter Lattermann ${ }^{*}$ \\ Makromolekulare Chemie I, Universität Bayreuth, D-95440 Bayreuth Germany
}

(Received 19 November 2004; published 5 May 2005)

\begin{abstract}
A thermoreversible (physical) gel consisting of a nematic liquid crystal mixed with a small quantity of a chiral organogelator is investigated in the planar configuration. The response of the system to an external electric field reveals multistability within a small hysteresis. The relaxation of the liquid crystal under this field is characterized by two different time scales: a fast one that is connected to the tilt of the director field, and a slow one that describes the reorientation of the chiral structure. In the first case, the relaxation is nonexponential and can be described by a Kohlrausch-Williams-Watts law with a stretching parameter of 0.5.
\end{abstract}

DOI: 10.1103/PhysRevE.71.051707

PACS number(s): 42.70.Df, 61.30.Gd, 64.70.Md, 83.80.Kn

\section{INTRODUCTION}

Today's liquid crystal displays are based on the Fréedericksz transition in a twisted nematic cell [1]: A nematic liquid crystal with a positive dielectric anisotropy $\epsilon_{a}>0$ is placed between two transparent electrodes which have been treated in such a way that the preferred axis of the nematic phase (the director) is fixed in the plane of the electrodes (planar alignment). The two electrodes are rotated against each other by a certain angle (typically $90^{\circ}$ or $270^{\circ}$ ), leading to a twisted nematic structure. This cell is sandwiched between two polarizers such that light perpendicular to the electrodes is transmitted through the cell. When a sufficiently large voltage is applied to the electrodes, the Fréedericksz transition occurs, leading to an almost homeotropic configuration of the liquid crystal (the preferred axis is now perpendicular to the glass plates throughout the cell), so that, due to the crossed polarizers, light can no longer pass through it. The switching process is reasonably fast for display applications; however, it relies on the usage of polarizers which not only reduce the transmitted light intensity but also negatively influence the viewing angle.

Recently, cholesteric (or chiral nematic) liquid crystals have also been used in electro-optically active elements [2]. A cholesteric mesophase or $N^{*}$ phase is the chiral version of the nematic phase $N$. It forms a spontaneous helical superstructure with a twist axis perpendicular to the director. The structure is periodic along the helical axis with a period of half the pitch, where the pitch is the distance over which the director completes a full $360^{\circ}$ rotation. Between two parallel electrodes, three typical cholesteric configurations can be observed $[3,4]$ (see also Fig. 3 below). In the planar state, the chiral nematic director is oriented parallel to the glass plates, so that the helical axes are perpendicular to the electrodes. Including defects, the so-called oily streak texture results. On

\footnotetext{
*Electronic address: wolfgang.schoepf@uni-bayreuth.de

†Electronic address: ingo.rehberg@uni-bayreuth.de

${ }^{\ddagger}$ Electronic address: guenter.lattermann@uni-bayreuth.de
}

the other hand, the helical axes may be oriented more or less parallel to the electrodes which, including defects, leads to the so-called fingerprint or focal-conic texture. ${ }^{1}$ As a third possibility, the homeotropic state may be realized, where the helical structure is destroyed and the director is parallel to the applied field [5].

The main interest has focused on displays that are based on the switching between two of these three configurations. In the planar configuration, a chiral nematic liquid crystal reflects circularly polarized light of one wavelength only, which is related to the pitch [6]. A sufficiently large electric field causes distortion and unwinding of the helices, leading to a homeotropic nematic structure [4], which is transparent for all polarizations of the incident light. Thus, a display which is based on the Bragg reflection of a single wavelength of light may be realized by switching between the planar and the homeotropic states. Another display technique utilizes the fact that the planar state appears transparent for wavelengths different from the Bragg reflections, while the fingerprint state is intrinsically nontransparent [7]. For both display techniques, no polarizers are needed so that the brightness of the display is higher and a wider viewing angle is achieved.

The transition from the planar to the fingerprint configuration for a cholesteric liquid crystal with positive dielectric anisotropy $\epsilon_{a}$ can be induced by applying an electric field. The direct inverse transition is much more difficult: depending on the pitch, the fingerprint state may remain stable for several days. Only for a large pitch does this relaxation time decrease to hours or even minutes, when the pitch becomes comparable to the cell thickness [8]. Thus, in most cases one effectively finds bistability with both states being possible at

\footnotetext{
${ }^{1}$ There is some confusion in the literature about the name of this texture. It has been regularly referred to as the storage mode, focalconic texture, polygonal texture, or fingerprint texture [39]. Some authors use the names focal-conic texture or polygonal texture for a more general mode with the fingerprint texture being a special case [4]. For simplicity, we use the name fingerprint texture throughout this paper.
} 
zero electric field, depending on the history of the probe. However, the material can be switched back to the planar state when coming from the homeotropic configuration, which is induced by using a voltage much higher than the one necessary for the fingerprint state [9].

Based on this planar-to-fingerprint transition is the socalled bistable cholesteric texture (BCT) display, which has been investigated in some detail $[7,10]$. In order to make it feasible for applications, however, some problems became apparent: small variations in the pressure or the temperature can dramatically change the pitch, thus varying the characteristics of the display. A successful attempt to avoid these problems was obtained with the development of the polymerstabilized cholesteric texture (PSCT), where the liquid crystal is mixed with less than $10 \%$ of a polymer [11-15]. The polymer forms a covalently cross-linked network stabilizing the different switching states of the liquid crystal layer and thus the cholesteric pitch, thereby minimizing the temperature sensitivity.

A further problem is the difficulty to switch from the fingerprint to the planar state. Using PSCT, the relaxation time can be reduced so that displays can be achieved without bistability. Here, the fingerprint state is only stable when the voltage is switched on so that the planar state is recovered at zero field [11]. The planar configuration is now stabilized by means of the covalently cross-linked gel. This mode is particularly attractive because it offers an excellent clear state at zero voltage, a large viewing angle, and a simple facility to switch from one configuration to the other [13].

Another possibility to solve the drawbacks of the BCT was given by Janssen et al. who presented an example of a BCT thermoreversible (physical) cholesteric gel [16]. Here, a cholesteric liquid crystalline mixture is gelified by a physical network instead of the covalently cross-linked polymer network as in the PSCT. The chiral nematic $N^{*}$ mesophase is obtained by mixing a chiral substance with nematic liquid crystals [17].

A second method to achieve a cholesteric liquid crystalline organogel was mentioned by Guan and Zhao [18]. A chiral organogelator can act as a chiral dopant for a nematic phase, when at higher temperatures and low concentrations the gelator is still dissolved in the nematic phase. Another approach to a cholesteric liquid crystal organogel was described by Moriyama et al. [19]. Here, the transformation of a nematic organogel to a liquid cholesteric phase, induced by uv irradiation of the trans-cis isomerization of a chiral organogelator, was observed. After the reisomerization to the trans configuration, either by keeping at room temperature or by irradiation with weak visible light, a stable cholesteric gel is formed.

In this paper, we present a thermoreversible gel, which consists of a nematic liquid crystal and a chiral gelator (see Fig. 1), inducing a cholesteric gel. In this system, the gelation temperature is lower than the clearing temperature, contrary to the system described in Ref. [18]. The resulting cholesteric gel may be used for a nonbistable display. This gel shows first the planar state and, upon applying an electric field, the fingerprint texture, and finally the homeotropic state. We perform a quantitative study in terms of amplitude, wavelength, and relaxation time near the threshold of the (a)

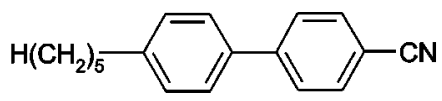

(b)

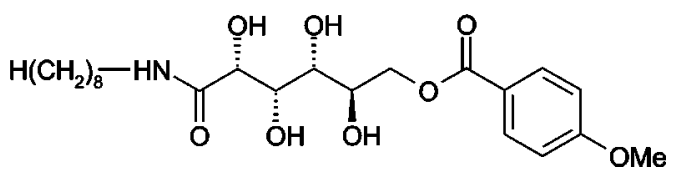

FIG. 1. (a) Compound 1: nematic liquid chrystal 4-cyano(4'-pentyl)biphenyl (5CB). (b) Compound 2: chiral gelator $N$ - $n$-octyl- $D$-gluconamid-6-(4-methoxybenzoate).

planar-to-fingerprint transition which may be useful for further theoretical investigations. Another goal is to characterize those properties which are important for display applications.

\section{EXPERIMENTAL SETUP}

We use one of the most investigated liquid crystals, 4-cyano-(4'-pentyl)biphenyl [5CB; compound 1; see Fig. 1(a)], which was obtained from Merck. 5CB is nematic at room temperature with a nematic-isotropic transition at $35^{\circ} \mathrm{C}$. It has a positive dielectric anisotropy of $\epsilon_{a}=11.8$, which results in a Fréedericksz threshold voltage of $U_{F}^{5 \mathrm{CB}}$ $=0.78 \mathrm{~V}$ and is thus well suited for our investigations.

The gelator $N$-n-octyl- $D$-gluconamid-6-(4-methoxybenzoate) [compound 2, see Fig. 1(b)] has previously been synthesized. It is a chiral, low-molecular-weight gelator based on a modified gluconamide derivative, which can gelify liquid crystals at very low concentrations [20]. The gelator molecules self-assemble through intermolecular hydrogen bonding building up rodlike structures (so-called cochleates) that interweave to form a homogeneous, thermoreversible physical network. The gelator itself acts as a chiral substance which induces the cholesteric phase into the nematic liquid crystal.

The liquid crystal physical gel was prepared by mixing $5 \mathrm{CB}$ and the gelator in a closed vial, using a concentration of $0.5 \mathrm{~mol} \%$ of the gelator. The vial was heated to $130^{\circ} \mathrm{C}$ and kept at this temperature until the gelator is fully dissolved into the isotropic liquid crystal. The solution is then cooled to $70{ }^{\circ} \mathrm{C}$ with a rate of $1 \mathrm{~K} / \mathrm{min}$. The temperature remains constant for $72 \mathrm{~h}$ to achieve a proper formation of the network. After this annealing time, the gelled system was allowed to cool down to room temperature with a cooling rate of $1 \mathrm{~K} / \mathrm{min}$. The chiral nematic phase appears at $35^{\circ} \mathrm{C}$.

We use the standard experimental setup as described, e.g., in Ref. [21]. The empty cells are commercially available. ${ }^{2}$ They consist of two parallel, transparent electrodes [glass plates with an indium tin oxide (ITO) layer on the inside] which are separated by spacers. The ITO surfaces of the electrodes are coated with a polymer and rubbed in one direction in order to produce a planar alignment. When this probe is filled with a nematic material, the director is aligned with the rub direction in the layer plane. This direction defines the $x$ axis, with the $y$ axis being perpendicular to $x$ in

\footnotetext{
${ }^{2}$ E.H.C. Co. Ltd., 1164 Hino, Hino-shi, Tokyo, Japan 191.
} 

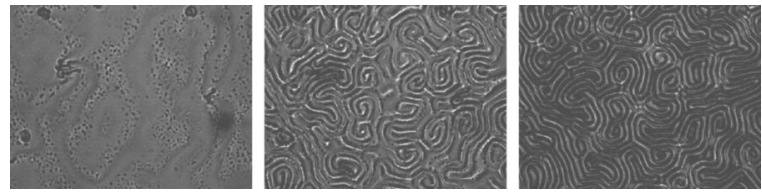

FIG. 2. The initial planar state at $f=2000 \mathrm{~Hz}$ for a small voltage $\left[U_{\mathrm{rms}}=0.12 \mathrm{~V}(\mathrm{left})\right]$. When increasing the voltage, the pattern develops into the fingerprint texture $\left[U_{\mathrm{rms}}=1.25\right.$ (middle) and $1.40 \mathrm{~V}$ (right)]. The height and width of the images are 0.42 and $0.55 \mathrm{~mm}$, respectively.

the layer plane. The thickness of the cell $d=25 \pm 1 \mu \mathrm{m}$ is determined by the spacer which separates the two glass plates in the $z$ direction.

The empty cell was held at $130{ }^{\circ} \mathrm{C}$ and filled by capillary action at this temperature, which is well above the gel-sol transition. In order to remove flow-induced defects which may have formed during filling, the system was kept another $10 \mathrm{~min}$ at this temperature before it was cooled to room temperature by the same annealing procedure as described above for the gel preparation. Finally, the cell was sealed. After further $24 \mathrm{~h}$ of maturing time, the measurements began, which were all performed at a temperature of $22{ }^{\circ} \mathrm{C}$ with a long-term stability of $\pm 5 \mathrm{mK}$.

A sinusoidal ac voltage $U(t)=U_{\text {rms }} \sqrt{2} \cos (2 \pi f t)$ is applied across the electrodes by means of a wave form generator. Due to the weakness of the physical network, the involved voltages are similar to those used for comparable, pure cholesteric liquid crystals. The cell is illuminated by a lightemitting diode with a wavelength of $\lambda=660 \mathrm{~nm}$. We observe the appearing patterns under a polarizing transmission microscope with the polarizer parallel to the $x$ direction and record them with a charge-couple device (CCD) camera connected to a frame grabber card. The images have a physical size of $552 \times 417 \mu \mathrm{m}^{2}$ and are digitized with a spatial resolution of $640 \times 484$ pixels into 256 gray scale values at a rate of 60 images per second. The patterns are visualized using the shadowgraph method [22]. The main feature here is the transformation of the spatially modulated refractive index, caused by the deflection of the director angle, into quantitative light intensity information resulting from geometrical optics.

\section{STATIC PROPERTIES}

\section{A. Qualitative overview}

We first give a qualitative picture of the primary instabilities which occur when the gel is subjected to an electrical voltage (Fig. 2). At zero voltage, the texture is characterized by weak scattering due to some defects, so that we consider this state as transparent. The initial pattern is the planar state, where the helical axes are perpendicular to the electrodes (Fig. 2, left image). The Bragg reflection was not observed, because the typical helix pitch in the material $(\approx 27 \mu \mathrm{m}$, see later) is much larger than the wavelength of light. When increasing the voltage to about $1 \mathrm{~V}$, the planar state is replaced by a new pattern. The material is now scattering for all polarizations of the incident light, so that we consider this

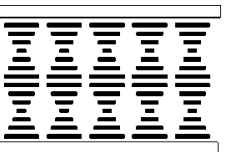

(a)

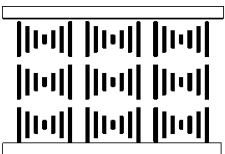

(b)

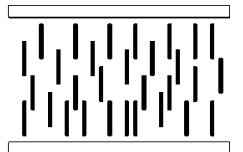

(c)
FIG. 3. Schematic arrangement of the liquid crystal director and the helical axes for the various states. The voltage is increased from left to right. (a) Planar director. (b) Helical axes parallel to the surface, fingerprint texture. (c) Helical axes unwound, director perpendicular to the surface, homeotropic state.

a scattering state (Fig. 2, middle and right images).

We identify this new pattern as the fingerprint texture, which may be considered a special case of the focal-conic texture $[3,4]$. The focal-conic texture consists of many domains, where within one domain the helical axes are parallel to each other, so that any orientation of the helices is present somewhere in the cell. If the helical axes are all parallel to the electrodes, the pattern is often called the fingerprint texture, which represents an isotropic state of curved parallel stripes. ${ }^{3}$ Due to the presence of the gel, we cannot be sure that the helices are all truly parallel to the electrodes, so that we may deal with a soft case of the focal-conic structure. Nevertheless, we will refer to this state as the fingerprint texture, as we do not measure a great variation of the pitch in our cell, as would be expected for the focal-conic structure.

When increasing the voltage in our system, the fingerprint texture gradually appears in an inhomogeneous form (middle image of Fig. 2) until it reaches its final state (right image). A further increase of the voltage to about $4 \mathrm{~V}$ leads to the so-called transient planar state, which is a rather complicated state somewhere between the fingerprint and the homeotropic configurations [23]. When increasing the voltage to about $7 \mathrm{~V}$, the structure develops entirely into the homeotropic state, where the helices have been unwound and the director is perpendicular to the plates (not shown here). A schematic picture of the various states is given in Fig. 3 .

It turns out that the initial planar state cannot easily be recovered directly from the homeotropic state, even if the voltage is switched off. There seems to be a slow relaxation process which may have a very long time scale, presumably of months. We assume that the gel network has been modified at high voltages and that the new configuration is now responsible for the slow relaxation with respect to a "normal" field-induced nematic-cholesteric transition [24,25]. However, when switching off the voltage already in the fingerprint state, the network-forming chiral gelator particles apparently stabilize the planar state, so that a quick relaxation back into the initial planar configuration is possible. Therefore, we focus our investigation on the transition between the planar state and the fingerprint texture and avoid the occurrence of the homeotropic state.

\section{B. Quantitative measurements}

The onset of the primary instability is measured in two steps. First, we start from the planar configuration and in-

\footnotetext{
${ }^{3}$ Sometimes, the name fingerprint texture is reserved for the case when in addition the helical axes are all parallel to each other, so that an anisotropic state of straight parallel lines occurs [6].
} 


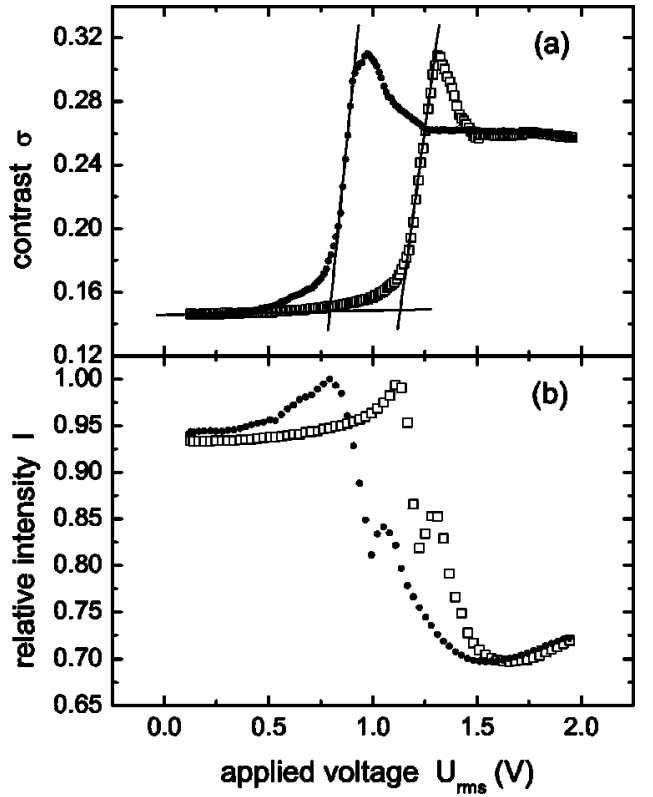

FIG. 4. Onset of the planar-fingerprint transition. The open squares indicate increasing and the solid circles decreasing voltages. The frequency of the electric field was fixed at $2000 \mathrm{~Hz}$. (a) Contrast $\sigma$ as a function of the applied voltage. The waiting time between voltage steps was $20 \mathrm{~s}$. (b) Transmitted intensity using the birefringence technique. The waiting time between voltage steps was $30 \mathrm{~s}$.

crease the voltage by small quantities for a fixed frequency. After each voltage step, we wait at least $20 \mathrm{~s}$ before a shadowgraph image is taken. This waiting time is much longer than the typical director relaxation time of the liquid crystal in such cells $\left(\tau_{d} \approx 1 \mathrm{~s}\right)$ and is usually sufficient for the system to relax in similar experiments [26]. Because the fingerprint texture is characterized by a large contrast (see Fig. 2), we define a suitable measure $\sigma$ for this contrast, which is used as an order parameter for the instability. $\sigma$ is essentially the root mean square of the intensity distribution of the picture, normalized by the maximum mean intensity $I_{\max }$ of all measurements:

$$
\sigma=\sqrt{\frac{\sum_{i=1}^{N}\left(I_{i}-\bar{I}\right)^{2}}{N I_{\max }^{2}}} .
$$

$I_{i}$ is the intensity of an individual pixel, $\bar{I}$ is the mean intensity of an image, and $N$ is the number of pixels per image. The second measurement is performed immediately after the first one by starting from the fingerprint configuration and then decreasing the voltage by the same small steps. The same waiting time is used, and the images are analyzed in the same way as above. The result of this measurement is shown in Fig. 4(a), where open squares refer to increasing and solid circles to decreasing the voltage. The critical voltage for the onset of the fingerprint texture is determined by the first measurement and yields $U_{\mathrm{rms}}^{c}=1.14 \mathrm{~V}$. The voltage for the inverse transition from the fingerprint texture to the

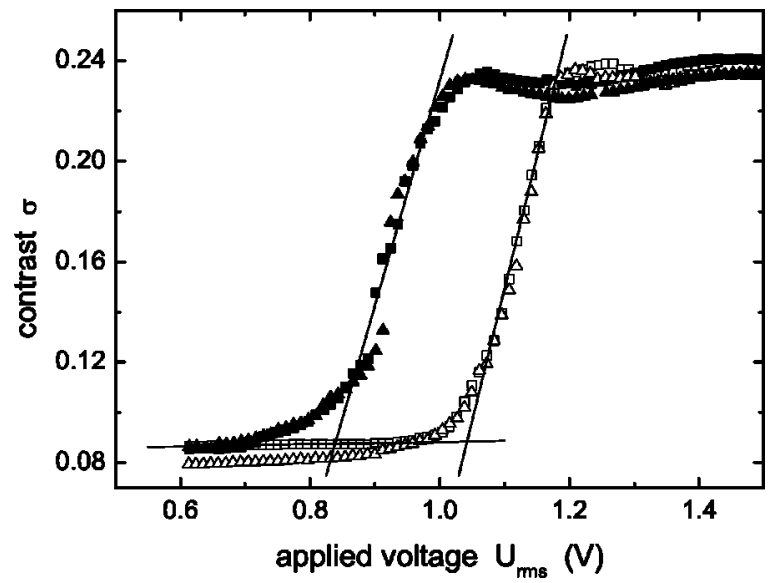

FIG. 5. Onset of the planar-fingerprint transition similar to Fig. 4(a), but with much longer waiting times between voltage steps. The triangles are for waiting times of $240 \mathrm{~s}$, while the squares are for $2700 \mathrm{~s}$. The open symbols indicate increasing and the solid symbols decreasing voltages.

planar alignment yields $U_{\mathrm{rms}}=0.78 \mathrm{~V}$; thus we find a hysteresis of $0.36 \mathrm{~V}$.

Measurements with longer waiting times (240 and 2700 s) have also been performed and are presented in Fig. 5. For the critical voltage, we find in this case $U_{\mathrm{rms}}^{c}=1.04 \mathrm{~V}$ and for the inverse transition $U_{\mathrm{rms}}=0.84 \mathrm{~V}$, obviously leading to a smaller hysteresis than observed in Fig. 4(a). On the other hand, the results for the two measurements shown in Fig. 5 are almost identical, so that we can conclude that even longer waiting times will not weaken the hysteresis further.

The important observation here is that within this hysteresis, we do not simply find bistability but rather multistability, as all the measurement points between the planar state and the fingerprint texture are individually stable. This feature is reminiscent of ferromagnetic behavior and may be explained in a similar way: the gel network forms chambers or domains which all have slightly different equilibrium preferences with respect to the orientation of the helical axes. The relatively small size of the hysteresis has to be compared to the case of those pure cholesteric liquid crystals, where bistability is observed at the planar-to-fingerprint transition [8].

In order to obtain a complementary characterization, we perform a further measurement of the instability using the birefringence technique $[1,27]$. Here, the probe is placed between two crossed polarizers, both of which are at an angle of $45^{\circ}$ with respect to the rub direction of the electrodes. This is the typical setup for measuring the Fréedericksz transition in nematic liquid crystals, where a change of the director orientation is translated into a change of the transmitted light intensity. Thus, the analog of the Fréedericksz transition should be observed with this method, i.e., the transition from a planar to a homeotropic configuration. Again, we perform the measurement for increasing and decreasing voltages, with a waiting time of $30 \mathrm{~s}$ between measurements. The results are shown in Fig. 4(b). The same hysteretic behavior as above is observed. It can be seen that the maxima of the transmitted intensities occur at the same voltages that were 


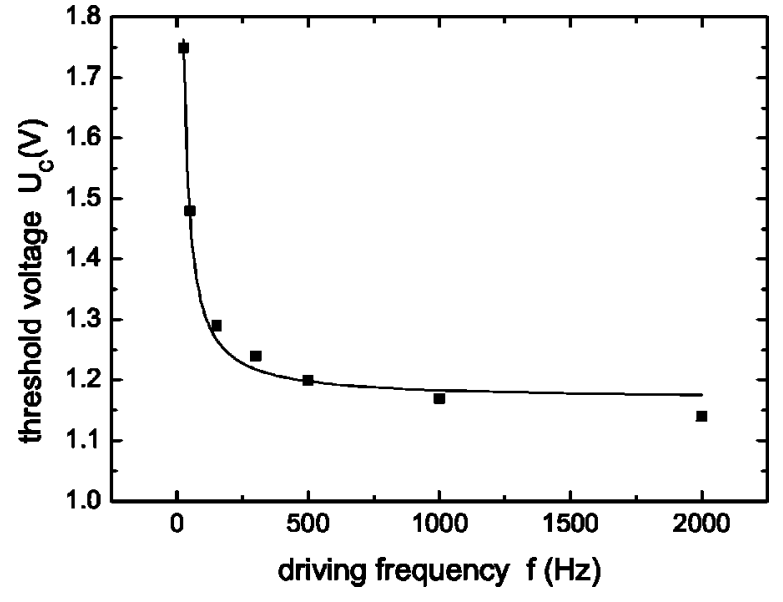

FIG. 6. Dependence of the threshold voltage of the planarfingerprint instability on the driving frequency. The line is only a guide to the eye.

obtained for the transition between the planar and the fingerprint states, for both increasing and decreasing voltages. The strong variation of the intensity beyond this maximum is due to the strong reorientation of the director during the transition. A reorientation of the director may be observed already before the transition, as it is indicated by the small intensity variation for increasing voltage in Fig. 4(b).

Remarkably, we observe a dependence of the critical voltage on the frequency of the applied field, which is shown in Fig. 6. The critical voltage increases toward small frequencies and seems to approach an asymptotic value for high frequencies. We propose that a hydrodynamic flow induced by charges, similar to the Carr-Helfrich mechanism [28,29], may hinder the reorientation of the helical axes. For high frequencies, such charge-induced effects are less important, and from the measurements we conclude that they can be neglected for frequencies higher than about $1000 \mathrm{~Hz}$.

In order to derive the pitch $p_{0}$ of our cholesteric material, we perform a statistic of the local wave vector $\vec{k}$ of the fingerprint pattern using the method presented in Ref. [30]: We approximate the light intensity of the fingerprint texture in a small area as $I(\vec{x})=A(\vec{x}) \cos [\phi(\vec{x})]$, where the local wave vector is $\vec{k}(\vec{x}) \equiv \vec{\nabla} \phi(\vec{x})$. If we assume that variations of $A(\vec{x})$ and $\vec{k}(\vec{x})$ are small compared to variations of $\phi(\vec{x})$, the components of the wave vector can be approximated in each point of the image by using simple partial derivates: $\left|k_{x}\right|^{2}$ $=-\partial_{x}^{2} I(\vec{x}) / I(\vec{x})$. A typical histogram of the modulus $k$ of the wave vector is presented in Fig. 7, which can be interpreted as a probability density for the wave number. For the fingerprint texture, the pitch can be determined as twice the wavelength $\lambda=2 \pi / k$ of the pattern [3]. From the measurement shown in Fig. 7 we find $p_{0}=27.4 \mu \mathrm{m}$. When changing the voltage or the frequency of the electric field, we could not find any variations of this value.

The critical voltage for our material $\left(U_{C}=1.04 \mathrm{~V}\right)$ is much smaller than the corresponding values for chemically cross-linked gels $\left(U_{C} \sim 10-30 \mathrm{~V}\right)[11,13]$. This was to be expected, since our physical network is weaker than a chemical network. Thus, a display based on this gel could be operated with more convenient voltages.

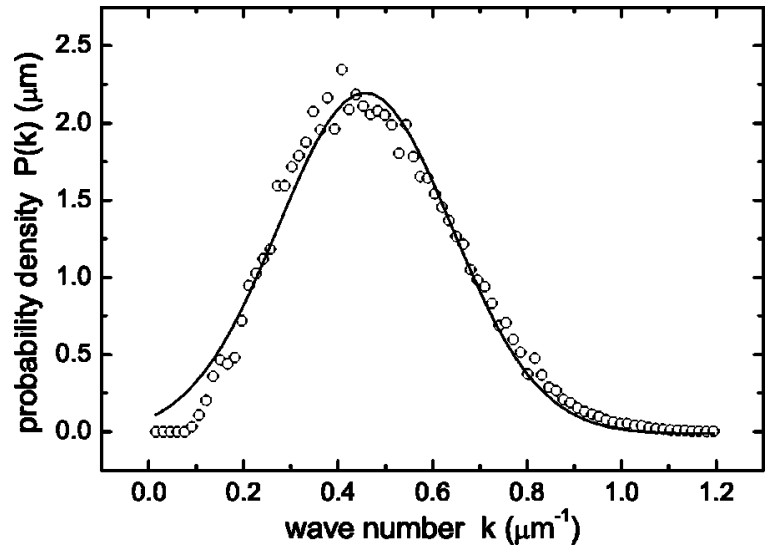

FIG. 7. Probability density of the local wave number of the fingerprint texture. The line is the fit of a Gaussian curve to determine the maximum at $k=0.46 \mu \mathrm{m}^{-1}$. The voltage for this measurement was $U_{\text {rms }}=1.50 \mathrm{~V}$ at $2000 \mathrm{~Hz}$.

We can compare the threshold voltage to the one obtained for a pure cholesteric liquid crystal, which for the planar-tofingerprint transition is given by $[5,31]$

$$
U_{\mathrm{PF}}=\left[\frac{k_{11} \pi^{2}}{\epsilon_{0} \epsilon_{a}}+\frac{4 k_{33} \pi^{2}}{\epsilon_{0} \epsilon_{a}}\left(\frac{d}{p_{0}}\right)^{2}\right]^{1 / 2} .
$$

Here, $k_{11}$ and $k_{33}$ are the splay and twist elastic constants of the liquid crystal. Using the values for $5 \mathrm{CB}$ and the pitch measured above, we find $U_{\mathrm{PF}}=1.99 \mathrm{~V}$, which is quite different from the value that we measured. However, the condition for the validity of this equation is not met in our experiment. ${ }^{4}$ On the other hand, we can also compare our measurement to the theoretical value for the Fréedericksz transition in a twisted nematic liquid crystal, which is given by [31]

$$
U_{\mathrm{FT}}=\left[\frac{k_{11} \pi^{2}}{\epsilon_{0} \epsilon_{a}}+\frac{4\left(k_{33}-2 k_{22}\right) \pi^{2}}{\epsilon_{0} \epsilon_{a}}\left(\frac{d}{p_{0}}\right)^{2}\right]^{1 / 2} \text {. }
$$

In this case, we find $U_{\mathrm{FT}}=1.18 \mathrm{~V}$, which is much closer to our value. It can be argued that the gel network imposes a twist on the otherwise nematic liquid crystal, so that the behavior should be better described by Eq. (3). We suggest that the gel network induces the chiral phase in a way similar to the one proposed by Berardi et al., where the cholesteric phase results from the contact of the nematic liquid crystal with a chiral surface [32].

\section{DYNAMIC RESPONSE}

\section{A. Evolution of the fingerprint pattern}

In order to investigate the dynamic behavior of the fingerprint pattern, we evaluate the step response of our system, i.e., when the applied voltage is suddenly changed. For this purpose, the frequency is kept constant at $2000 \mathrm{~Hz}$ and the voltage is changed either from subcritical to supercritical or vice versa. We follow the temporal evolution of the pattern

\footnotetext{
${ }^{4}$ Expression (2) is valid either for $k_{22}>k_{33}$, or for $k_{22} \leqslant k_{33}$ and $d / p_{0}<\left[k_{22} / 3\left(k_{33}-k_{22}\right)\right]$.
} 


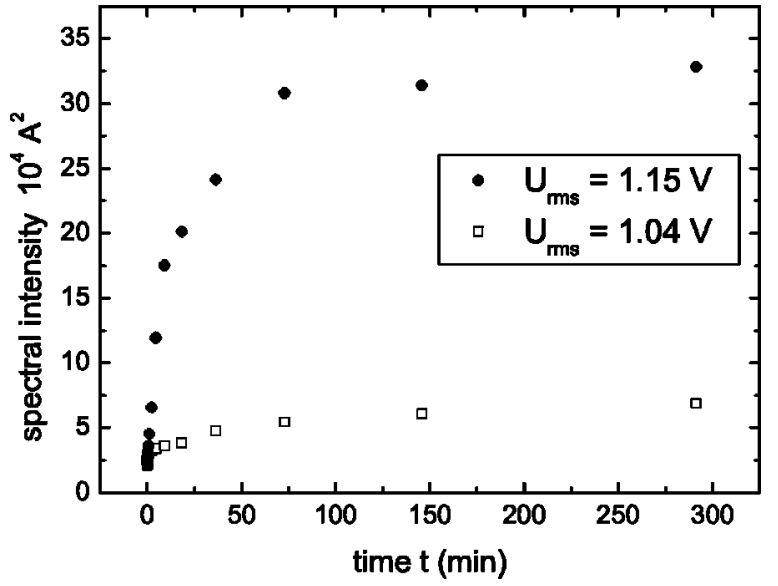

FIG. 8. Temporal evolution of the amplitude of the fingerprint pattern in the so-called slow regime. The evolution is shown for two different voltage steps at $2000 \mathrm{~Hz}$.

with our CCD camera. Typically, the camera is started upon the change of the voltage with images being sampled at a rate of 10 full frames per second. The developing pattern is characterized by calculating the two-dimensional Fourier transform $A(\vec{k})$ of each picture and its square, the spatial power spectrum $S(\vec{k})$ [33]. Since the pattern is isotropic, we average $S(\vec{k})$ over the polar angle to get $S(k)$. We find the intensity $A^{2}$ of our pattern as the integral over the first peak of the function $S(k)$. All results are normalized by the first Fourier mode $A_{0}^{2}=N^{2} I_{\max }^{2}$.

The intensity $A^{2}$ is primarily a measure for the development of the macroscopic quantities of the fingerprint pattern, for which the orientations of the helices are responsible. Since the tilting process of the director is very fast and does not strongly affect the configuration of the helices, we believe that the relaxation times measured here correspond to the reconfiguration of the helices. The fast tilting process of the director, which is reminiscent of the Fréedericksz transition, cannot be observed with these experiments and will be discussed below in Sec. IV B.

In a first set of experiments, we jump from $0.12 \mathrm{~V}$ to a supercritical value. For each voltage step, a time series of images of the evolving fingerprint pattern is obtained. Thus, we can calculate the spectral intensity $A^{2}$ of the pattern as a function of time for each voltage step. It turns out that we can distinguish between two different temporal regimes when we switch on the voltage. If the final voltage is not too far above the fingerprint threshold, we observe a very slow regime, where the amplitude relaxes over several hours onto its equilibrium value, which is shown in Fig. 8. Obviously, the steady-state value of the amplitude is only reached after about 1-2 h. For steps toward higher voltages (in our case to above $1.45 \mathrm{~V}$ ), we find a much faster regime, where the amplitude of the fingerprint pattern relaxes within a matter of seconds, as shown in Fig. 9. Here, the final value of the amplitude is achieved already after about $30 \mathrm{~s}$.

Assuming an exponential increase after applying a voltage step, we fit a function of the form $A^{2}(t)=A_{0}^{2} \exp (2 t / \tau)$ to the data obtained for the amplitude. This is shown by the solid line in Fig. 9 and yields the grow rate $1 / \tau$ for this

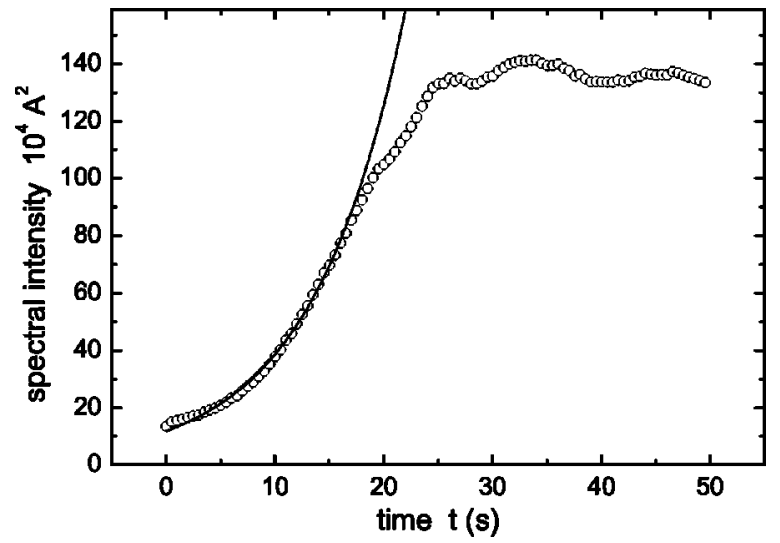

FIG. 9. Temporal evolution of the amplitude of the fingerprint pattern in the so-called fast regime. The evolution is shown for switching the voltage from 0.12 to $1.50 \mathrm{~V}$ at $2000 \mathrm{~Hz}$. The solid line is the fit of an exponential function to the experimental data.

particular experiment. Repeating this procedure for each voltage step, we obtain the growth rate as a function of the target voltage for all our steps. The result is presented by the open squares in Fig. 12 below and will be discussed in detail in Sec. IV C.

In a second set of experiments, we jump from $1.50 \mathrm{~V}$ to a subcritical value. We perform the same procedure as above, i.e., for each voltage step, we calculate the spectral intensity $A^{2}$ of the dissolving pattern as a function of time. We assume an exponential decay of the form $A^{2}=A_{0}^{2} \exp (2 t / \tau)+C^{2}$, where the offset accounts for experimental imperfections. If we fit this function to the data obtained for each voltage step, we get the decay rate as a function of the target voltage for all our steps. The result is presented by the solid squares in Fig. 12. The decay rates are comparable to the values obtained for the fast regime of the growing patterns, and the measured times are again of the order of seconds.

\section{B. Tilting of the director (Fréedericksz transition)}

A further series of experiments is performed, using the birefringence technique. As discussed in Sec. III B above, a change in the director tilt is translated into a variation of the transmitted light intensity, which allows for a detection of the Fréedericksz transition. For small voltage steps, the variation of the light intensity is proportional to the change of the director angle. Starting in the planar configuration, we increase the voltage in steps of $0.06 \mathrm{~V}$. Immediately after the change, we follow the temporal evolution of the light intensity with our CCD camera at a rate of 60 frames per second. After waiting for two minutes in order to allow the system to relax onto its new equilibrium state, the next voltage step is initiated. A well-developed fingerprint state is reached at $U_{\mathrm{rms}}=1.77 \mathrm{~V}$. Here we reverse the procedure and decrease the voltage in the same small steps of $0.06 \mathrm{~V}$. Examples of the temporal evolution of the light intensity are shown in Figs. 10(a) and 10(b) for increasing and decreasing voltages, respectively.

We tried to fit a simple exponential law (increasing or decreasing) to data such as presented in Fig. 10, but found 


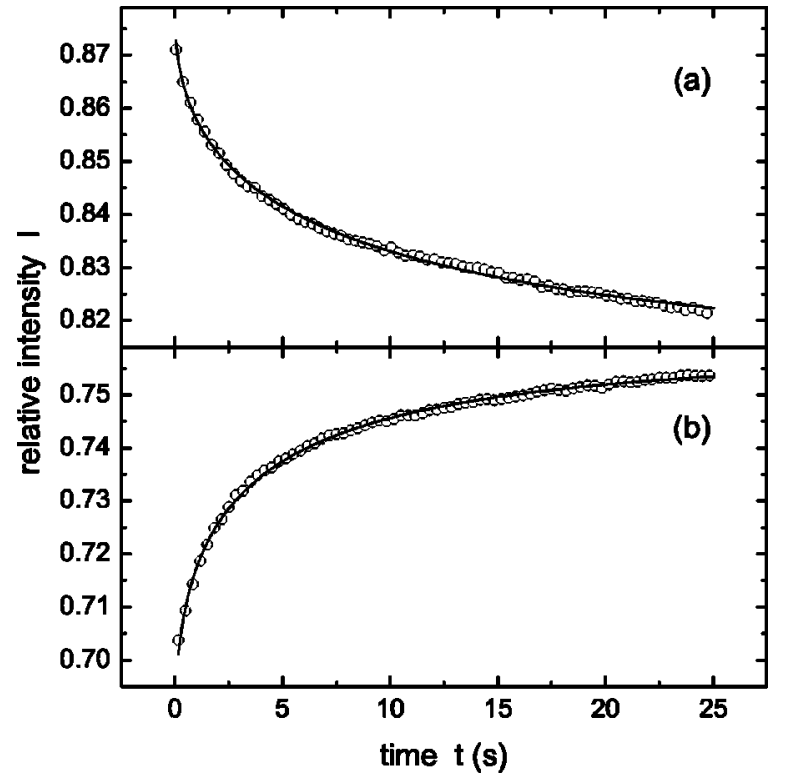

FIG. 10. Temporal evolution of the transmitted light intensity after changing the voltage, using the birefringence technique. (a) The applied voltage is increased from $U_{\text {rms }}=1.19$ to $1.25 \mathrm{~V}$. (b) The voltage is decreased from 1.08 to $1.02 \mathrm{~V}$. The solid lines are fits of a stretched exponential Kohlrausch-Williams-Watt law as discussed in the text.

systematic deviations. On the other hand, the existence of domains in our liquid crystal, which are due the gelator and have been discussed in Sec. III B, may lead to a nonsimple exponential behavior of the director field. A good candidate to describe the relaxation of the director is the streched exponential Kohlrausch-Williams-Watt (KKW) law [34], which characterizes the relaxation in complex systems, such as, e.g., the decay of the remanent magnetization in glasses [35] or the coarse-graining of magnetic domains [36]. Moreover, Tsang et al. have shown how the interaction between different domains in a system of oscillators induces a similar behavior to the one observed here [37]. Thus, we fit the KKW function

$$
I(t)=I_{\infty}+\left(I_{0}-I_{\infty}\right) e^{-(t / \tau)^{\beta}}
$$

to our experimental data. $I_{0}$ and $I_{\infty}$ are the starting and equilibrium values of the transmitted light intensity, $1 / \tau$ is the characteristic growth rate, and $\beta$ is the fractional Kohlrausch exponent. Such fits are shown by the lines in Fig. 10, which obviously describe the data quite well. The results for $\beta$ are presented in Fig. 11. $\beta$ seems to vary randomly around some mean value, but shows no clear dependence on the voltage. In order to have a well-reproducible procedure for determining the growth rates, an optimal value for $\beta$ was obtained by minimizing the deviation between the fits and the experimental data for all curves. Subsequently, we fitted the data again, but now with $\beta$ kept constant at this value of $\beta=0.48$. The growth rates resulting from this procedure are shown in Fig. 12 for increasing (open circles) and decreasing (solid circles) voltage.

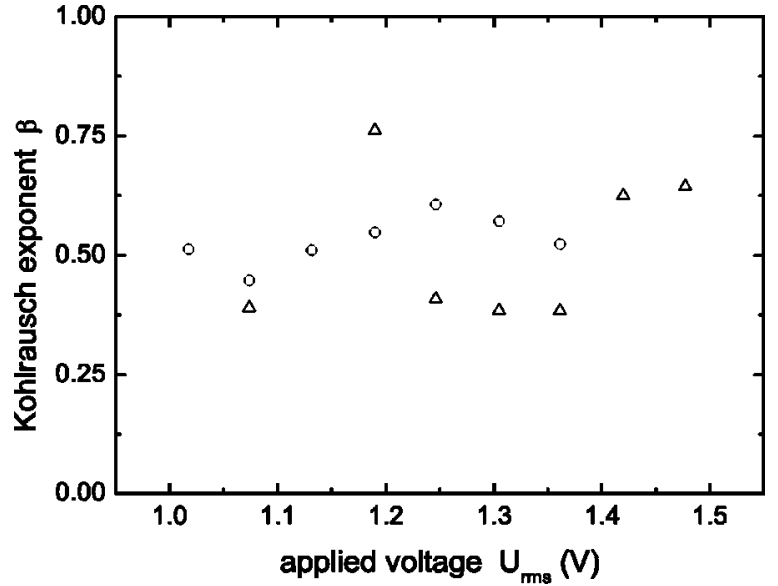

FIG. 11. Kohlrausch exponent $\beta$ as a function of the applied voltage $U_{\text {rms }}$ for increasing (triangles) and decreasing (circles) voltage. No clear dependence on the voltage can be deduced.

\section{Discussion of the growth rates}

From theoretical considerations, we expect a behavior like $1 / \tau \propto\left(U^{2}-U_{0}^{2}\right)$; thus in Fig. 12 the growth rates are plotted against the squared voltage with the solid lines being linear fits. The respective slopes are $0.18\left(\mathrm{~V}^{2} \mathrm{~s}\right)^{-1}$ for the growth rate of the fingerprint pattern (open squares) and $0.24\left(\mathrm{~V}^{2} \mathrm{~s}\right)^{-1}$ for the decay rate (solid squares). For the relaxation times measured with the birefringence technique, we find $1.10\left(\mathrm{~V}^{2} \mathrm{~s}\right)^{-1}$ for increasing (open circles) and $1.03\left(\mathrm{~V}^{2} \mathrm{~s}\right)^{-1}$ for decreasing (solid circles) voltage. In the latter case, we find a similar hysteresis to the one obtained with the static measurements (see Sec. III B). The crossover

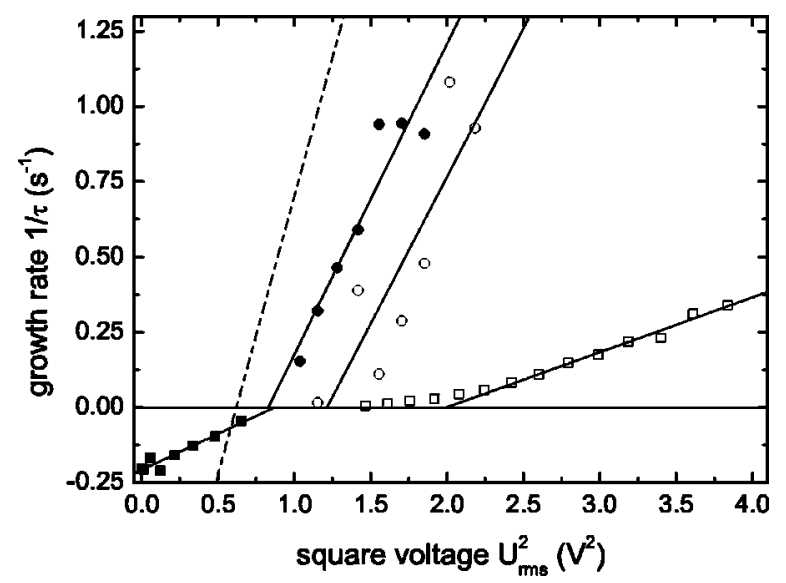

FIG. 12. Growth rates $1 / \tau$ for the various measurements presented in Secs. IV A and IV B. The open squares correspond to voltage steps from subcritial $(0.12 \mathrm{~V})$ to supercritical, while the solid squares are for steps from supercritical $(1.50 \mathrm{~V})$ to subcritical, as described in Sec. IV A. Both measurements refer to the evolution of the fingerprint pattern. The open and solid circles correspond to the measurements described in Sec. IV B. Here, the voltage has been increased (open) and decreased (solid) in small steps, so that the tilting of the director can be observed. The solid lines represent linear fits to the data, while the theoretical growth rate for pure $5 \mathrm{CB}$ is shown by the dashed line as a comparison. 
with the zero-growth-rate line occurs at $U_{\mathrm{rms}}=1.10$ and 0.91 $\mathrm{V}$, respectively. As a comparison, the theoretical growth rate for the Fréedericksz transition in pure 5CB is shown by the dashed line which has a slope of $1.85\left(\mathrm{~V}^{2} \mathrm{~s}\right)^{-1}$ [38].

It can be seen from the open circles in Fig. 12 that the occurrence of the Fréedericksz transition is shifted toward higher values when compared to pure $5 \mathrm{CB}$. This is not surprising, as the introduction of chirality leads to a higher threshold value [see Eqs. (2) and (3)]. This shift was also noticed in the static measurements discussed in Sec. III B.

We will now consider the various growth rates presented in Fig. 12 and try to explain their relative magnitudes. The decay rates of the fingerprint pattern shown by the solid squares are much slower than those for the Fréedericskz transition in the pure material, which is expected as both transitions are quite different. The Fréedericksz transition is well understood in terms of the equilibrium between the elastic, the dielectric, and the viscous torques exerted on the director. On the other hand, the occurrence of the fingerprint pattern is much more complicated. For simplicity, we first consider only the relaxation of the fingerprint texture in a pure cholesteric liquid crystal (no gel) after switching off the voltage. The system is initially metastable and thus remains in the fingerprint configuration. Only for a large pitch and when the anchoring of the director at the glass plates is sufficiently strong can the system relax into the planar state. This transition is not simple, possibly involving a quick transition into the nematic phase before the planar chiral texture is reached. Indeed, the measured relaxation times are of the order of minutes to days. A more detailed study was provided by Luban et al. [39].

In order to evaluate the influence of the gelator, we compare our results with similar, but slightly different experiments. It turns out that the relaxation times of the fingerprint pattern after switching off the voltage $\left(\tau_{\text {off }} \sim 15 \mathrm{~s}\right)$ are faster than those for pure cholesteric liquid crystals $\left(\tau_{\text {off }} \geq 1 \mathrm{~min}\right.$ $[8,39])$, but much slower than those observed for covalently cross-linked gels $\left(\tau_{\text {off }} \sim 5 \mathrm{~ms}\right.$ [11] $)$ where the gelator acts strongly. We suggest that due to the domains created by our gelator, the typical transition time is lowered as compared to a pure cholesteric liquid crystal where no domains are present. When decreasing the voltage from the fingerprint regime, we expect the planar state to reoccur first in those places where domains already favor this state. Such domains act as nucleation points and thus favor the transition between the two metastable states, so that the planar pattern grows from these places into the remainder of the cell.

The growth rates for the transition from the planar to the fingerprint state shown by the open squares have similar values to the decay rates just discussed. We speculate that a similar process happens as above, so that this state also nucleates from domains which already favor the fingerprint configuration. Again, the time scale is faster than for the pure cholesteric case. This behavior is observed for steps to higher voltages; however, we have no explanation for the slow regime that occurs when the voltage is increased to values not too far above the transition point, as discussed in Sec. IV A.
The time scales for tilting the liquid crystal director are represented by the open and solid circles in Fig. 12. They have to be compared to the Fréedericksz transition in pure 5CB (dashed line), which turns out to be about 1.7 times faster than the tilting mode measured in our experiments. Kwok and Liao calculated this rate to be the same for cholesteric and nematic liquid crystals [40], but no experimental evidence was found to substantiate this claim. We suggest that the gel network will not only slow down this mode, but also create domains which further change the relaxation behavior of the system from the typical exponential behavior to the stretched exponential law observed here. The growth rates are essentially the same for increasing (open circles) and decreasing (solid circles) voltage.

\section{SUMMARY AND CONCLUSION}

We have doped a nematic liquid crystal with $0.5 \mathrm{~mol} \%$ of a chiral gelator and in this way obtained a thermoreversible cholesteric gel. Measurements of the electric-field-induced reorientation of the director field reveal a hysteretic switching process accompanied by multistability. This behavior is unusual for liquid crystals, but reminiscent of ferromagnetism and presumably due to the formation of domains with different director orientations caused by the gel network.

The orientation of the liquid crystal under an external electric field can be characterized by two different relaxation processes: a fast one, which is connected to the tilt of the director field, and a slow one, namely, the reorientation of the chiral structure on a length scale of about $25 \mu \mathrm{m}$.

While the dynamics of the Fréedericksz transition in a pure nematic liquid crystal can be described by a simple exponential relaxation, the gel reacts in a fashion which is more typical for disordered systems, namely, a stretched exponential relaxation. The stretching parameter seems to be close to 0.5 , a result that presently lacks any explanation.

The slower relaxation time is related to the reorientation of the helices and has to be considered a nuisance when thinking in terms of display applications. Although these cholesteric structures do not necessarily have a strong impact on the optical contrast of the display, we conclude that they should be avoided in such applications. It must be stated that the origin of the cholesteric structure in our system is not yet completely understood; however, the morphological characterization of our cholesteric gel is currently in progress. Future investigations will deal with the influence of the kind of gelator, of different gelator morphologies, and of the kind of liquid crystal on the switching dynamics.

\section{ACKNOWLEDGMENTS}

We wish to thank Werner Pesch and Helmut Brand for helpful discussions and suggestions. A.d.L. has been supported by a grant from the Deutsche Forschungsgemeinschaft (Grant No. GRK 698). O.L. and G.L. gratefully acknowledge the financial support of the Deutsche Forschungsgemeinschaft (Grant No. La 662/10-1,2) and the supply of 5CB by Merck. 
[1] L. M. Blinov, Electro-Optical and Magneto-Optical Properties of Liquid Crystals (Wiley, New York, 1983).

[2] G. P. Crawford and S. Žumer, Liquid Crystals in Complex Geometries (Taylor \& Francis, London, 1996).

[3] I. Dierking, Textures of Liquid Crystals (Wiley-VCH, Weinheim, 2003), pp. 54-74.

[4] G. Chilaya, in Chirality in Liquid Crystals, edited by H.-S. Kitzerow and C. Bahr (Springer, New York, 2001), p. 163.

[5] H. A. van Sprang and J. L. M. van de Venne, J. Appl. Phys. 57, 175 (1985).

[6] P. G. de Gennes and J. Prost, The Physics of Liquid Crystals (Clarendon Press, Oxford, 1993).

[7] D. K. Yang, J. W. Doane, Z. Yaniv, and J. Glasser, Appl. Phys. Lett. 64, 1905 (1994).

[8] J. P. Hulin, Appl. Phys. Lett. 21, 455 (1972).

[9] J. V. Gandhi, X. D. Mi, and D. K. Yang, Phys. Rev. E 57, 6761 (1998).

[10] X. Y. Huang, D. K. Yang, and J. W. Doane, Appl. Phys. Lett. 67, 1211 (1995)

[11] D. K. Yang, L. C. Chien, and J. W. Doane, Appl. Phys. Lett. 60, 3102 (1992)

[12] M. H. Lu, J. Appl. Phys. 81, 1063 (1997).

[13] H. Ren and S. T. Wu, J. Appl. Phys. 92, 797 (2002).

[14] R. A. M. Hikmet and H. Kemperman, Liq. Cryst. 26, 1645 (1999).

[15] R. A. M. Hikmet and R. Polesso, Adv. Mater. (Weinheim, Ger.) 14, 502 (2002).

[16] R. H. C. Janssen, J. P. Teunissen, S. J. Picken, C. W. M. Bastiaansen, D. J. Broer, T. A. Tervoort, and P. Smith, Jpn. J. Appl. Phys., Part 1 40, 2372 (2001).

[17] G. W. Gray, Thermotropic Liquid Crystals (John Wiley \& Sons, New York, 1987).

[18] L. Guan and Y. Zhao, J. Mater. Chem. 11, 1339 (2001).

[19] M. Moriyama, N. Mizoshita, T. Yokota, K. Kishimoto, and T. Kato, Adv. Mater. (Weinheim, Ger.) 15, 1335 (2003).
[20] O. Lafuente, Ph.D. thesis, Universität Bayreuth, 2004.

[21] I. Rehberg, B. L. Winkler, M. de la Torre Juárez, S. Rasenat, and W. Schöpf, Adv. Solid State Phys. 29, 35 (1989).

[22] S. Rasenat, G. Hartung, B. L. Winkler, and I. Rehberg, Exp. Fluids 7, 412 (1989).

[23] J. E. Anderson, P. Watson, and P. J. Bos, Liq. Cryst. 28, 945 (2001).

[24] J. J. Wysocki, J. Adams, and W. Haas, Phys. Rev. Lett. 20, 1024 (1968).

[25] F. J. Kahn, Phys. Rev. Lett. 24, 209 (1970).

[26] A. de Lózar, T. Bock, M. Müller, W. Schöpf, and I. Rehberg, New J. Phys. 5, 63 (2003).

[27] L. Bata, A. Buka, and I. Janossy, Solid State Commun. 15, 647 (1974)

[28] E. F. Carr, Mol. Cryst. Liq. Cryst. 7, 253 (1969).

[29] W. Helfrich, J. Chem. Phys. 51, 4092 (1969).

[30] D. A. Egolf, I. V. Melnikov, and E. Bodenschatz, Phys. Rev. Lett. 80, 3228 (1998).

[31] F. M. Leslie, Mol. Cryst. Liq. Cryst. 12, 57 (1970).

[32] R. Berardi, H. G. Kuball, R. Memmer, and C. Zannoni, J. Chem. Soc., Faraday Trans. 94, 1229 (1998).

[33] Y. Hu, W. Pesch, G. Ahlers, and R. E. Ecke, Phys. Rev. E 58, 5821 (1998).

[34] G. Williams and D. C. Watts, Trans. Faraday Soc. 66, 80 (1970).

[35] G. Kriza and G. Mihály, Phys. Rev. Lett. 56, 2529 (1986).

[36] B. Reimann, R. Richter, and I. Rehberg, Phys. Rev. E 65, 031504 (2002).

[37] K. Y. Tsang and K. L. Ngai, Phys. Rev. E 54, R3067 (1996).

[38] P. Pieranski, F. Brochard, and E. Guyon, J. Phys. (Paris) 34, 35 (1973).

[39] M. Luban, S. Shtrikman, and J. Isaacson, Phys. Rev. A 15, 1211 (1977).

[40] S. K. Kwok and Y. Liao, J. Appl. Phys. 49, 3970 (1978). 